\title{
Embolia pulmonar aérea secundaria a colangiopancreatografía retrógrada endoscópica en una paciente con trasplante hepático. Reporte de caso
}

\section{Case report of a pulmonary air embolism secondary to endoscopic retrograde cholangiopancreatography in a liver transplant patient}

Gustavo Adolfo Reyes Medina, MD, ${ }^{1}$ Renzo Pinto Carta, MD, ${ }^{1}$ Fernando Sierra Arango, MD, ${ }^{2}$ Luis Felipe Cabrera Vargas, MD. ${ }^{3}$

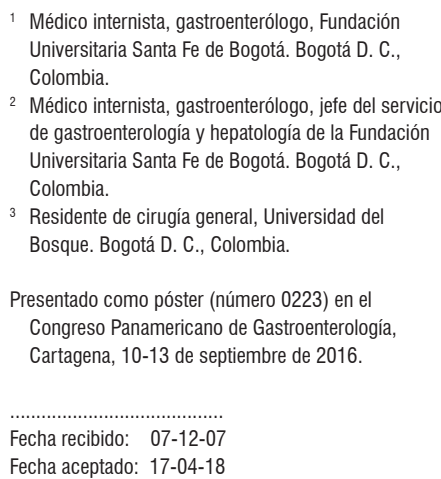

2 Médico internista, gastroenterólogo, jefe del servicio de gastroenterología y hepatología de la Fundación Universitaria Santa Fe de Bogotá. Bogotá D. C., Colombia.

${ }^{3}$ Residente de cirugía general, Universidad del Bosque. Bogotá D. C., Colombia.

Presentado como póster (número 0223) en el Congreso Panamericano de Gastroenterología, Cartagena, 10-13 de septiembre de 2016.

Fecha recibido: $\quad 07-12-07$

Fecha aceptado: 17-04-18

\begin{abstract}
Resumen
La colangiopancreatografía retrógrada endoscópica (CPRE) es uno de los recursos terapéuticos más importantes para el manejo de las complicaciones biliares del trasplante hepático. Sin embargo, se pueden presentar varias complicaciones: pancreatitis aguda, hemorragia, perforaciones, infecciones y eventos adversos cardiopulmonares. La embolia aérea es una complicación muy infrecuente, severa y potencialmente fatal. Se reporta un caso de embolia aérea post-CPRE en una mujer de 55 años con antecedente de trasplante hepático y estenosis de la anastomosis biliar. Se discute la presentación clínica, el diagnóstico, el tratamiento y los posibles mecanismos involucrados en esta complicación.

Palabras clave

Colangiopancreatografía retrógrada endoscópica (CPRE), complicaciones, embolia aérea, trasplante hepático.

Abstract

Endoscopic retrograde cholangiopancreatography (ERCP) is one of the most important therapeutic resources for management of biliary complications of liver transplantation. However, several complications including acute pancreatitis, hemorrhage, perforations, infections and cardiopulmonary adverse events can occur. Air embolisms occur very infrequently but are severe and potentially fatal complications. We report a case of post-ERCP embolism in a 55-year-old woman with a history of liver transplantation and stenosis of the biliary anastomosis. The clinical presentation, the diagnosis, the treatment and the possible mechanisms involved in this complication are discussed.
\end{abstract}

\section{Keywords}

Endoscopic retrograde cholangiopancreatography (ERCP), complications, air embolism, liver transplant.

\section{INTRODUCCIÓN}

La embolia aérea como complicación de un procedimiento endoscópico es una entidad extremadamente infrecuente, pero potencialmente fatal $(1,2)$. En una revisión sistemática publicada en el 2013, de 41 casos de embolia aérea secundaria a procedimientos endoscópicos reportados hasta ese momento en la literatura, la mayoría ( 26 casos) fue secundaria a colangiopancreatografía retrógrada endoscó- pica (CPRE) (1). Es causada por una comunicación directa entre el aire insuflado en la luz intestinal y los vasos sanguíneos de la pared intestinal y/o biliar, asociada con un gradiente de presión que favorece el paso del aire a la circulación sanguínea y a una mucosa intestinal o biliar alterada. El efecto fisiológico de la embolia aérea depende de la velocidad y del volumen del aire infundido a la circulación. Puede ser de tipo venoso o arterial y producir isquemia de cualquier órgano (1). Existen mecanismos para la embolia aérea pos-CPRE: 
la transección de las venas radiculares duodenales durante la esfinterotomía, la disección de aire intramural hasta la vena porta, la presencia de derivaciones o fístulas biliovenosas, de venas colaterales porto-cavas, el flujo de aire directamente hacia la vena hepática o hacia la vena cava inferior, el flujo retrógrado de aire hacia las venas cerebrales por vía de la vena cava superior y la inhabilidad de la circulación pulmonar para filtrar el émbolo aéreo (1-3).

\section{REPORTE DE CASO}

Mujer de 55 años, con trasplante hepático en 2007 por síndrome de Budd-Chiari, que desarrolló estenosis anastomótica biliar. Se manejó mediante CPRE durante 1 año (dilataciones neumáticas, inserción de prótesis biliares plásticas). Por recurrencia de la estenosis biliar se insertó un stent metálico autoexpandible (SMAE) completamente recubierto $(80 \mathrm{~mm}$ por $10 \mathrm{~mm}$ ). A los 4 meses consultó por dolor abdominal, se realizó una ecografía abdominal que mostró dilatación del colédoco $(20 \mathrm{~mm})$ con cálculos en su interior. Los paraclínicos mostraron elevación de la fosfatasa alcalina $(3 \mathrm{x})$ con bilirrubinas $\mathrm{y}$ transaminasas normales (fosfatasa alcalina: $290 \mathrm{UI} / \mathrm{L}$, valor normal: 32-91; aspartato-aminotransferasa [AST]: $25 \mathrm{U} / \mathrm{L}$, valor normal: 15-51; alanina- aminotransferasa [ALT]: $31 \mathrm{U} / \mathrm{L}$, valor normal: $17-63$; bilirrubina total: $0,9 \mathrm{mg} / \mathrm{dL}$, valor normal: 0,3-1,2; bilirrubina directa: $0,2 \mathrm{mg} / \mathrm{dL}$, valor normal: 0,1-0,5). Se realizó una CPRE bajo anestesia general. En la radiografía preliminar se apreció el stent biliar metálico y neumobilia proximal (Figura 1A). En la duodenoscopia se observó que el SMAE ha migrado proximalmente; se introdujo un balón extractor en su interior, se insufló, se traccionó, se expuso parcialmente su extremo distal en luz duodenal y se logró extraer con una pinza de cuerpo extraño. Al examinarlo, el SMAE tenía su lumen totalmente obstruido por cálculos y barro biliar.

La colangiografía mostró una vía biliar extrahepática dilatada (colédoco proximal de 18 milímetros de diámetro) y la anastomosis biliar sin estenosis (Figura 1B). Se instrumentó la vía biliar con balón, con extracción de cálculos residuales. En la placa final había importante neumobilia que comprometía además los pequeños conductos biliares proximales intrahepáticos (Figura 1C). En el posoperatorio inmediato (paciente aún intubada) se documentó un ritmo de taquicardia ventricular seguido de un trazo de actividad eléctrica sin pulso; presentó desaturación, hipotensión, cianosis y marcada ingurgitación yugular, se iniciaron medidas de reanimación, y el retorno a circulación espontanea se obtuvo después de 2 minutos, con administración concomitante de $1 \mathrm{mg}$ de adrenalina, $300 \mathrm{mg}$ de amiodarona, $60 \mathrm{meq}$ de bicarbonato e infusión de norepinefrina $(0,25 \mu \mathrm{g} / \mathrm{k} / \mathrm{m})$. Se accedió a la vía venosa central subclavia derecha.

Se tomó una ecografía FAST que descartó taponamiento cardíaco, pero evidenció múltiples burbujas de aire en la vena cava; se trasladó inmediatamente a angiografía por TAC (bajo sospecha de embolia aérea), que mostró aire en la vena cava intrahepática (Figura 2) y émbolo aéreo
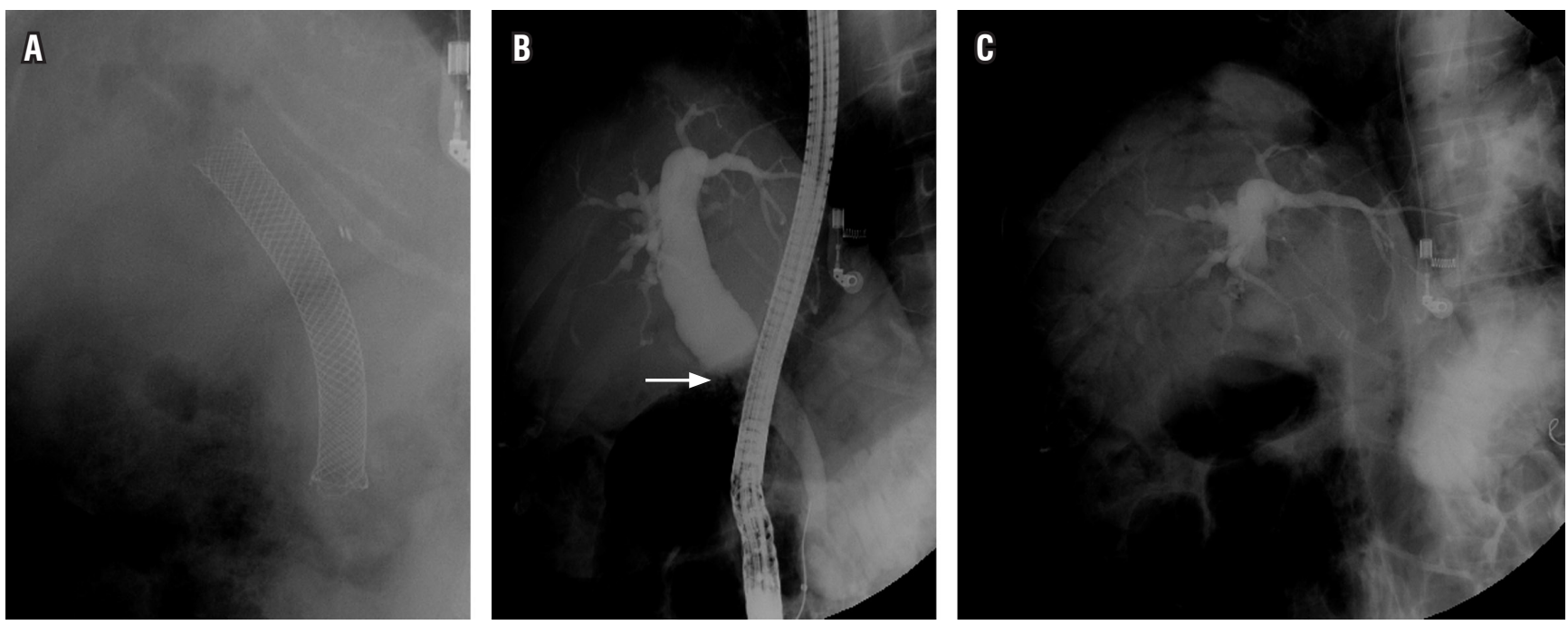

Figura 1. A. Radiografía preliminar, con SMAE y neumobilia en la vía biliar proximal, por encima del borde proximal del stent. B. Colangiograma después del retiro de la prótesis biliar. Colédoco proximal dilatado, anastomosis biliar (flecha) sin estenosis. C. Radiografía al final del procedimiento, con medio de contraste residual en vía biliar proximal y extensa neumobilia en la vía biliar intrahepática. 
en una de las arterias pulmonares subsegmentarias del segmento apical del lóbulo inferior derecho (Figura 3). Se trasladó a la paciente a la unidad de cuidados intensivos (UCI), se tomó ecocardiograma transtorácico que mostró colapso de la cava inferior, hallazgo esperado en un estado posreanimación, sin disfunción ventricular izquierda. La paciente evolucionó hacia la mejoría, se extubó al segundo día y se dio de alta a los 4 días sin complicaciones adicionales ni secuelas.

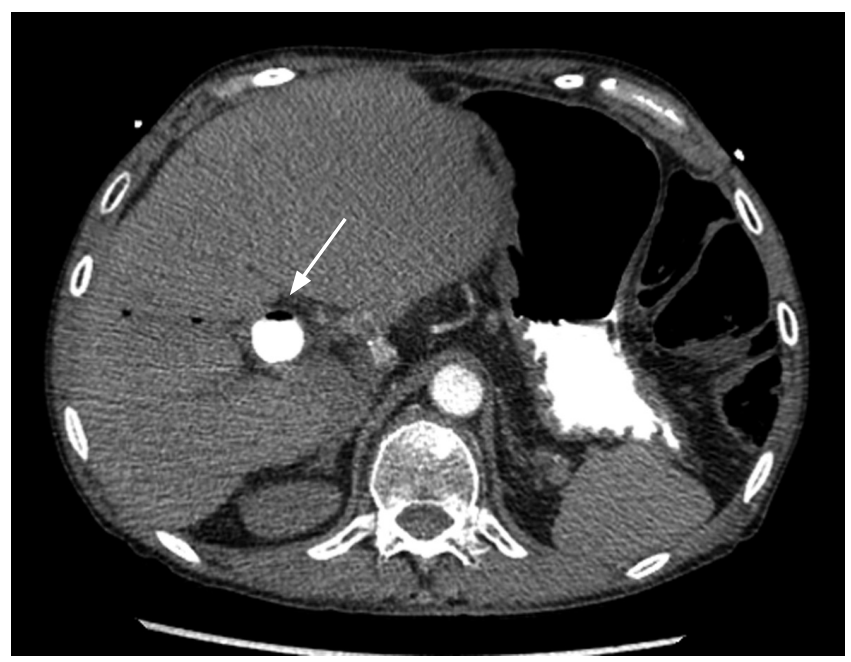

Figura 2. Angio-TAC. Aire en la vena cava inferior intrahepática (flecha).

\section{DISCUSIÓN}

La embolia aérea se caracteriza por inestabilidad hemodinámica debido a la llegada del aire al ventrículo derecho con aumento de la presión en la arteria pulmonar, obstrucción del flujo de salida del ventrículo derecho y compromiso del retorno venoso pulmonar al corazón izquierdo, lo que altera la precarga, con disminución del gasto cardíaco, colapso cardiovascular e insuficiencia vascular de órganos como el cerebro $(1,2)$. Se pueden generar arritmias, hipotensión, isquemia miocárdica, insuficiencia cardíaca derecha aguda, hipoxia, cianosis, ingurgitación yugular, desviación ocular, midriasis, alteración de la conciencia, hipertonicidad, hemiparesia, edema cerebral, coma y muerte $(3,4)$.

Generalmente, los síntomas de embolia aérea durante una CPRE se presentan cuando el paciente se reacomoda en posición de decúbito supino al final del procedimiento $(1,5)$. La mayoría de los síntomas son inespecíficos y se requiere una alta sospecha clínica para realizar un diagnóstico correcto e iniciar el manejo adecuado (6).

Esta complicación está más frecuentemente relacionada con la CPRE, pero puede presentarse en cualquier otro procedimiento endoscópico (5). Como factores de riesgo asociados se encuentran: intervención previa endoscópica o quirúrgica del árbol biliar, derivación portosistémica intrahepática transyugular (TIPS), trauma cerrado o penetrante del hígado, procesos inflamatorios del tracto digestivo (pileflebitis, absceso hepático, enfermedad inflamatoria intestinal, enterocolitis necrotizante, isquemia mesentérica), fístula gastrointestinal postoperatoria, neoplasias gastrointestinales, atresia de las vías biliares, procedimientos intervencionistas (colangioscopia, esfinterotomía biliar, drenaje hepático percutáneo, stent metálico biliar), biopsia hepática, insuflación de aire a altos volúmenes y/o alta presión, sitio del procedimiento por encima el nivel del corazón y el uso de óxido nitroso $(1,2)$. La insuflación con dióxido de carbono $\left(\mathrm{CO}_{2}\right)$ en lugar de aire disminuye el riesgo de la embolia aérea, debido a que el $\mathrm{CO}_{2}$ se absorbe más rápida y fácilmente. La monitorización cardíaca con Doppler precordial durante el procedimiento puede detectar tempranamente aire en el corazón y en la vasculatura pulmonar, antes de que los síntomas aparezcan $(5,6)$.

Una de las características más comunes de los pacientes que desarrollan embolia aérea pos-CPRE es el antecedente de intervenciones previas (endoscópicas o quirúrgicas) en el árbol biliar (5). En el caso que se reporta existen varios factores asociados que pudieron actuar conjuntamente para el desarrollo de la embolia aérea: múltiples intervenciones previas en el tracto biliar (trasplante hepático con anastomosis biliar termino-terminal, varias CPRE para el manejo de estenosis biliar, la inserción de un SMAE) y la presencia de cálculos biliares y de un SMAE ocluido en su lumen, con el consiguiente proceso inflamatorio en las paredes del conducto biliar (4). Debido a la migración proximal del stent biliar y a las maniobras necesarias para su retiro, se produjo trauma de la mucosa biliar y de la papila duodenal durante la extracción del stent (en una mucosa previamente inflamada). Esto favorece la exposición de los vasos sanguíneos mucosos y submucosos al contacto con el aire y su posterior difusión intravascular $(1,2)$.

La extracción de una prótesis obstruida y la instrumentación de la vía biliar con balón, asociado con una comunicación amplia entre la vía biliar y la luz duodenal por la papilotomía previa, produce una presión negativa en el árbol biliar que facilita el paso rápido del aire del duodeno hacia la vía biliar intrahepática, lo cual se demuestra en la placa final de la CPRE (importante neumobilia que compromete los pequeños conductos biliares intrahepáticos en forma generalizada). Este aire en la vía biliar (en abundante cantidad y rápidamente ingresado) puede difundir fácilmente hacia la circulación sistémica (cavidades cardíacas derechas) a través de las venas hepáticas, lo cual pudo constituir otro mecanismo adicional para la embolia aérea (3).

El diagnóstico de esta entidad se dificulta ya que el aire puede ser rápidamente absorbido de la circulación mien- 

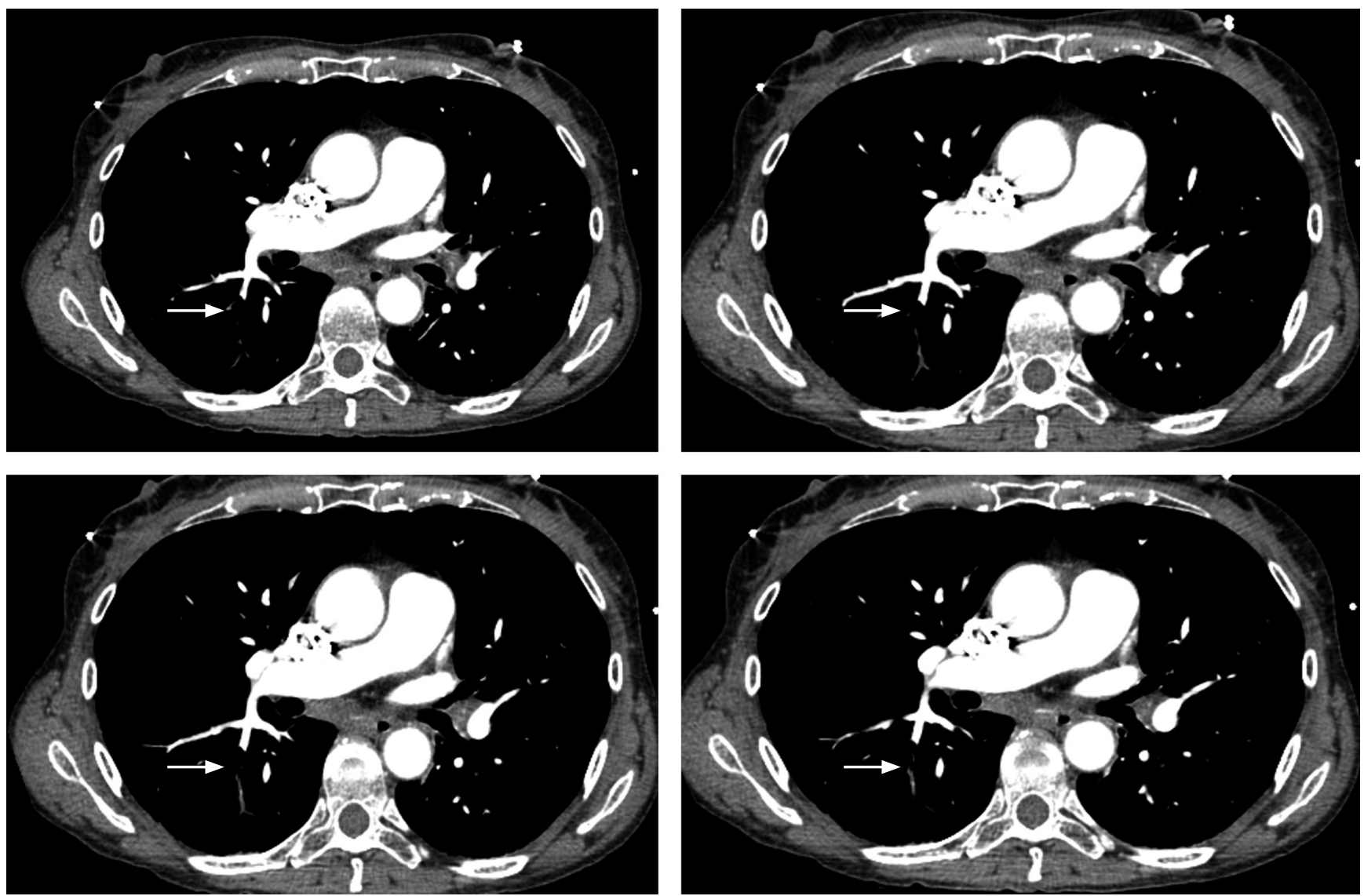

Figura 3. Émbolo aéreo en la arteria pulmonar subsegmentaria, segmento apical del lóbulo inferior derecho (flechas).

tras se están realizando los exámenes diagnósticos. El ecocardiograma transtorácico o transesofágico se ha empleado para identificar la presencia de aire en el ventrículo derecho, con dilatación de este, e hipertensión de la arteria pulmonar; y permite descartar anomalías de la contractilidad cardíaca $(1,7)$. La angiografía por TAC puede detectar con mayor sensibilidad un émbolo aéreo $(2,6)$.

En el caso presentado se realizó una ecografía FAST que demostró aire en la vena cava, lo que, junto con el cuadro clínico, sugirió el diagnóstico de una embolia aérea, que fue confirmada con una angiografía por TAC realizada inmediatamente después de la fase inicial de la reanimación cardiorrespiratoria.

El manejo se basa en sospechar la embolia aérea y mientras se confirma se deben realizar maniobras para disminuir el impacto fisiológico, tales como detener inmediatamente el procedimiento endoscópico, administrar oxígeno al 100 $\%$, iniciar solución salina en grandes volúmenes, colocar al paciente en posición de Trendelenburg y decúbito lateral izquierdo para minimizar la migración del aire al cerebro y suspender el óxido nitroso en caso de estar usándose (1,
4). Se puede realizar aspiración del aire a través de un catéter central e iniciar terapia con oxígeno hiperbárico, lo que reduce el tamaño de las burbujas, acelera la reabsorción del nitrógeno y disminuye el riesgo de isquemia $(1,2)$. Si se presenta un paro cardiorrespiratorio, se debe iniciar reanimación cardiopulmonar avanzada, la cual además disminuye el tamaño de las grandes burbujas y fuerza al aire a salir del ventrículo derecho hacia las venas pulmonares (1).

\section{CONCLUSIONES}

El embolismo aéreo pos-CPRE es una complicación infrecuente. Se deben tener en cuenta los factores de riesgo asociados con esta complicación. Ante la sospecha clínica se debe realizar un diagnóstico rápido e iniciar el tratamiento adecuado, ya que el diagnóstico tardío puede conllevar a una fatalidad.

\section{Fuentes de financiación}

Ninguna. 


\section{Conflictos de interés}

Los autores del artículo manifiestan no tener conflictos de interés.

\section{REFERENCIAS}

1. Donepudi S, Chavalitdhamrong D, Pu L, Draganov PV. Air embolism complicating gastrointestinal endoscopy: A systematic review. World J Gastrointest Endosc. 2013;5(8):35965. doi: 10.4253/wjge.v5.i8.359.

2. Kwon CI, Song SH, Hahm KB, Ko KH. Unusual complications related to endoscopic retrograde cholangiopancreatography and its endoscopic treatment. Clin Endosc. 2013;46(3):251-9. doi: 10.5946/ce.2013.46.3.251.

3. Cha ST, Kwon CI, Seon HG, Ko KH, Hong SP, Hwang SG, et al. Fatal biliary-systemic air embolism during endoscopic retrograde cholangiopancreatography: a case with multifocal liver abscesses and choledochoduodenostomy. Yonsei Med J. 2010;51(2):287-90. doi: 10.3349/ymj.2010.51.2.287.

4. Bisceglia M, Simeone A, Forlano R, Andriulli A, Pilotto A. Fatal systemic venous air embolism during endoscopic retrograde cholangiopancreatography. Adv Anat Pathol. 2009; 16(4):255-62. doi: 10.1097/PAP.0b013e3181aab793.

5. Green BT, Tendler DA. Cerebral air embolism during upper endoscopy: case report and review. Gastrointest Endosc. 2005;61(4):620-3.

6. Bechi A, Nucera MP, Olivotto I, Manetti R, Fabbri LP. Complete neurological recovery after systemic air embolism during endoscopic retrograde cholangiopancreatography. Minerva Anestesiol. 2012;78(5):622-5.

7. Rangappa P, Uhde B, Byard RW, Wurm A, Thomas PD. Fatal cerebral arterial gas embolism after endoscopic retrograde cholangiopancreatography. Indian J Crit Care Med. 2009;13(2):108-12. doi: 10.4103/0972-5229.56061. 